\title{
INTEGRATION FORMULAS TO EVALUATE FUNCTIONS OF RANDOM VARIABLES
}

\author{
Jianhua Zhou and Andrzej S. Nowak \\ Department of Civil Engineering, University of Michigan, Ann Arbor, MI 48109 (U.S.A.)
}

(Received December 15, 1987; accepted in revised form September 10, 1988)

Key words: reliability; numerical integration; functions; statistical moments; random variables; probability; statistical parameters.

\begin{abstract}
Structural reliability analysis often involves extensive integration of a function of multiple basic variables. Special techniques can be used to reduce this numerical effort. This paper develops integration formulas for computing the statistical parameters of a function of a random vector, in particular calculation of the first few moments. The formula is a numerical procedure using selected weights and points to estimate integrals. The points and weights are predetermined in the independent standard normal variable space. The sample points in basic variable space are then obtained by various transformations. The formulas were developed for the basic variables with various probability information known. The procedure is convenient to implement, and allows for a direct use of the available deterministic computer programs.
\end{abstract}

\section{INTRODUCTION}

Recently, there have been extensive new developments in reliability theory and its application to structural engineering. Especially noteworthy has been the work of Ang and Cornell [1], Galambos and Ravindra [2], and Ellingwood et al. [3] in the United States, Lind [4] in Canada, and Ditlevsen [5] and Rackwitz [6] in Europe. A more critical review on structural reliability methods can be found in the book by Madsen et al. [7].

In structural reliability analysis, load and resistance models play a very important role. The models are derived using survey data, tests, analysis and engineering judgement. Often the resistance or load is expressed as a function of multiple basic variables such as material properties, dimensions, geometries, frequency and magnitude of load, and so on. It is then necessary to evaluate the statistical parameters, particularly the first few moments, of these 
functions. Various numerical methods have been widely used, such as the Monte Carlo method [8], Latin Hypercube Sampling [9,10], and point estimates [11,12]. However, Monte Carlo simulation or Latin Hypercube Sampling require prohibitively large computational effort. although the number of simulations is independent of the number of basic variables. This paper develops a simple numerical integration procedure for computing the statistical parameters of a function of multiple random variables. The samples of basic variables are obtained by transforming a priori the selected points in standard normal space to basic variable space. The method is similar to point estimates, except that the points and weights are predetermined in standard normal space. The procedure is convenient to implement, and allows a direct use of the available deterministic computer programs.

\section{THEORETICAL DEVELOPMENT}

For a general function of a random vector $\boldsymbol{X}=\left(X_{1}, X_{2}, \ldots, X_{n}\right)$, that is $G(\boldsymbol{X})=G\left(X_{1}, X_{2}, \ldots, X_{n}\right)$

the exact $k$ th moment of $G, E\left[G^{k}(\boldsymbol{X})\right]$, may be obtained by evaluating the integral:

$E\left[G^{k}(\boldsymbol{X})\right]=\int_{-\infty}^{+\infty} \ldots \int_{-\infty}^{+\infty} f_{\boldsymbol{X}}\left(x_{1}, \ldots, x_{n}\right) G^{k}\left(x_{1}, \ldots, x_{n}\right) \mathrm{d} x_{1} \ldots \mathrm{d} x_{n}$

where $f_{X}\left(x_{1}, \ldots, x_{n}\right)$ is the joint probability density function (PDF) of the random vector $\boldsymbol{X}$. In many applications, however, the integration of eqn. (2) may be difficult to perform, and special numerical procedures are needed. Gorman [13] used the quadrature formulas to evaluate eqn. (2) for the special cases of normal and lognormal random variables. In this study numerical integration formulas were formulated for any jointly distributed random vector.

\section{Single standard normal variable}

Let $Z$ denote a single standard normal variable with the probability density function defined as

$f_{Z}(z)=\phi(z)=\frac{1}{\sqrt{2 \pi}} \exp \left(-\frac{1}{2} z^{2}\right)$

Substituting eqn. (3) into eqn. (2) and $z=\sqrt{2} u(\mathrm{~d} z=\sqrt{2} \mathrm{~d} u)$ yields

$$
\begin{aligned}
E\left[G^{k}(Z)\right] & =\int_{-\infty}^{+\infty} \phi(z) G^{k}(z) \mathrm{d} z \\
& =\frac{1}{\sqrt{\pi}} \int_{-\infty}^{+\infty} \exp \left(-u^{2}\right) G^{k}(\sqrt{2} u) \mathrm{d} u
\end{aligned}
$$

The integration of eqn. (4b) can be evaluated using the Gauss-Hermite formula [14]:

$\frac{1}{\sqrt{\pi}} \int_{-\infty}^{+\infty} \exp \left(-u^{2}\right) G^{k}(\sqrt{2} u) \mathrm{d} u \cong \frac{1}{\sqrt{\pi}} \sum_{j=1}^{m} \alpha_{j} G^{k}\left(\sqrt{2} u_{j}\right)$

where $\alpha_{j}$ and $u_{j}$ are the $j$ th weight factor and zero of the $(2 m-1)$ th order Hermite polynomial, respectively, which are widely available [14], and $m$ is the number of points considered. 


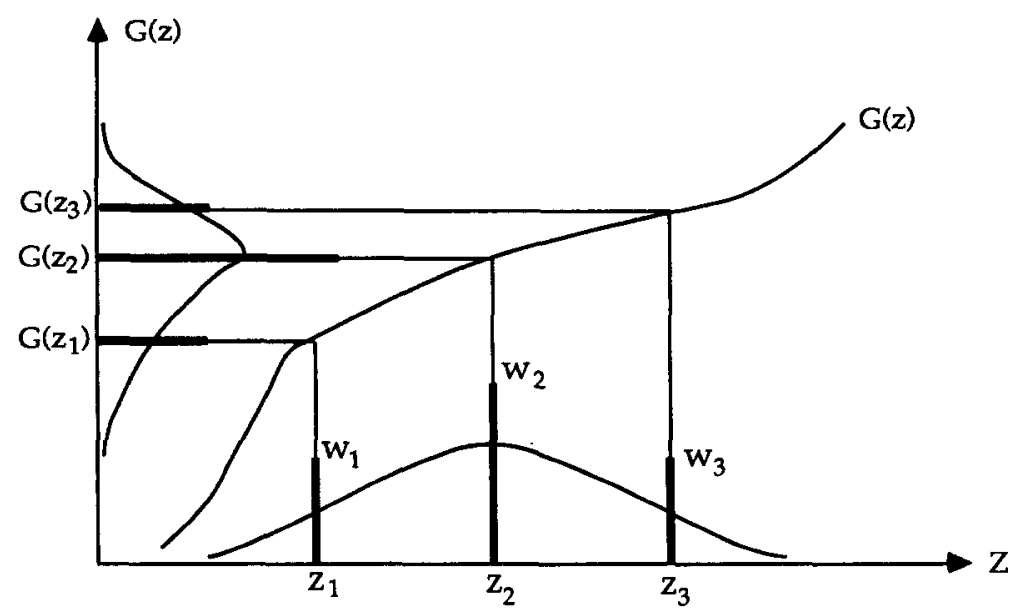

Fig. 1. Illustration of three-point integration.

Using $z_{j}=\sqrt{2} u_{j}$ and letting $w_{j}=\alpha_{j} / \sqrt{\pi}$, weight factors and points in standard normal space ( $Z$ space) are obtained. Thus eqn. (5) becomes

$\int_{-\infty}^{+\infty} \phi(z) G^{k}(z) \mathrm{d} z \cong \sum_{j=1}^{m} w_{j} G^{k}\left(z_{j}\right)$

Equation (6) is the integration formula for a single standard normal variable, and it is graphically shown in Fig. 1. When $G^{k}(z)$ is a polynomial of at most the $(2 m-1)$ th degree, eqn. (6) is exact. Typical normal points $z_{j}$ and the corresponding weights $w_{j}$ are given in Table 1 .

\section{Single non-normal variable}

Let $X$ denote a non-normal variable in eqn. (2) and make a marginal transformation [15]:

$X=F_{X}^{-1}(\Phi(z))$

where $\Phi(z)$ is the cumulative distribution function (CDF) of standard normal variable $Z$, and $F_{X}(x)$ is the cumulative distribution function of non-normal variable $X$. The transformation is graphically shown in Fig. 2. By this transformation the domain of the integration eqn. (2) is changed from $X$ space to the standard normal space:

$\int_{-\infty}^{+\infty} f_{X}(x) G^{k}(x) \mathrm{d} x=\int_{-\infty}^{+\infty} \phi(z) G^{k}\left(F_{X}^{-1}(\Phi(z))\right) \mathrm{d} z$

The right side of eqn. (8) can be evaluated using eqn. (6)

$\int_{-\infty}^{+\infty} \phi(z) G^{k}\left(F_{X}^{-1}(\Phi(z))\right) \mathrm{d} z \cong \sum_{j=1}^{m} w_{\mathrm{j}} G^{k}\left(F_{X}^{-1}\left(\Phi\left(z_{j}\right)\right)\right)$

That is, for a non-normal variable $X$, eqn. (2) can be calculated using the formula

$E\left[G^{k}(X)\right] \cong \sum_{j=1}^{m} w_{j} G^{k}\left(x_{j}\right)$ 


\section{TABLE 1}

Examples of points and weight factors for integration formulas

\begin{tabular}{lll}
\hline Number of points & Points & Weight factors \\
$m$ & $z_{1}$ & $w_{1}$ \\
\hline 1 & $z_{1}=0$ & $w_{1}=1$ \\
2 & $z_{1}=-1$ & $w_{1}=\frac{1}{2}$ \\
$z_{2}=+1$ & $w_{2}=\frac{1}{5}$ \\
& $z_{1}=-\sqrt{3}$ & $w_{1}=\frac{1}{6}$ \\
$z_{2}=0$ & $w_{2}=\frac{1}{6}$ \\
& $z_{3}=+\sqrt{3}$ & $w_{3}=\frac{1}{6}$ \\
& $z_{1}=-\sqrt{3+\sqrt{6}}$ & $w_{1}=(3-\sqrt{6}) / 12$ \\
& $z_{2}=-\sqrt{3-\sqrt{6}}$ & $w_{2}=(3+\sqrt{6}) / 12$ \\
& $z_{3}=+\sqrt{3-\sqrt{6}}$ & $w_{3}=(3+\sqrt{6}) / 12$ \\
& $z_{4}=+\sqrt{3+\sqrt{6}}$ & $w_{4}=(3-\sqrt{6}) / 12$ \\
& $z_{1}=-\sqrt{5+\sqrt{10}}$ & $w_{1}=(7-2 \sqrt{10}) / 60$ \\
5 & $z_{2}=-\sqrt{5-\sqrt{10}}$ & $w_{2}=(7+2 \sqrt{10}) / 60$ \\
& $z_{3}=0$ & $w_{3}=8 / 15$ \\
& $z_{4}=+\sqrt{5-\sqrt{10}}$ & $w_{4}=(7+2 \sqrt{10}) / 60$ \\
& $z_{5}=+\sqrt{5+\sqrt{10}}$ & $w_{5}=(7-2 \sqrt{10}) / 60$ \\
\hline
\end{tabular}

in which

$x_{j}=F_{X}^{-1}\left(\Phi\left(z_{j}\right)\right)$

where $z_{j}$ and $w_{j}$ are the points and weight factors associated with standard normal space. Equation (10) is the integration formula for a single non-normal variable.

\section{Multiple standard normal variables}

In many practical problems, reliability analysis often involves multiple random variables. In this study, the foregoing method has been generalized to a function of multiple variables. This

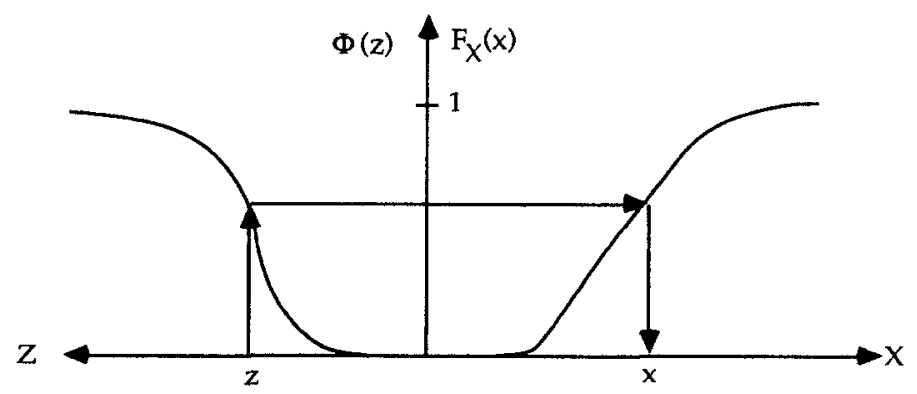

Fig. 2. Transformation of normal variable to non-normal variable. 
section describes the development of integration formulas for a function of independent standard normal variables. The following sections describe integration formulas for a function of multiple non-normal variables.

In the case of an independent standard normal vector $Z=\left(Z_{i}, Z_{2}, \ldots, Z_{n}\right)$ the joint probability density function is,

$f_{Z}\left(z_{i}, z_{2}, \ldots, z_{n}\right)=\phi\left(z_{1}\right) \phi\left(z_{2}\right) \ldots \phi\left(z_{n}\right)$

Equation (2) becomes

$E\left[G^{k}(Z)\right]=\int_{-\infty}^{+\infty} \ldots \int_{-\infty}^{+\infty} \phi\left(z_{1}\right) \ldots \phi\left(z_{n}\right) G^{k}\left(z_{1}, \ldots, z_{n}\right) \mathrm{d} z_{1} \ldots \mathrm{d} z_{n}$

Two approaches have been developed to evaluate above integration: the product integration formula and the non-product integration formula.

\section{Product integration formula}

The product formula is based on the factorization of $G$ into single-variable functions:

$G\left(Z_{1}, Z_{2}, \ldots, Z_{n}\right)=\prod_{i=1}^{n} G_{i}\left(Z_{i}\right)$

Such an operation is difficult since usually the function $G$ is given only implicitly as a function of $Z_{1}, Z_{2}, \ldots, Z_{n}$. Special techniques are required to approximately factorize $G\left(Z_{1}, \ldots, Z_{n}\right)$ into $G_{1}\left(Z_{1}\right) G_{2}\left(Z_{2}\right) \ldots G_{n}\left(Z_{n}\right)$. A method suggested by Rosenblueth [11] was modified for the current study.

Based on Rosenblueth's suggestion a function $G=G\left(Z_{1}, Z_{2}, \ldots, Z_{n}\right)$ is approximated by a function $H=H\left(Z_{1}, Z_{2}, \ldots, Z_{n}\right)$, which is defined by

$H=G_{0} \prod_{i=1}^{n} \frac{G_{i}^{*}}{G_{0}}=G_{0} \prod_{i=1}^{n} G_{i}\left(Z_{i}\right)$

where $G_{0}=G(0,0, \ldots, 0)$, i.e., the function evaluated at the zero means of variables $Z, G_{i}^{*}$ are the functions of $G$ computed as though $Z_{i}$ were the only random variable, and other variables were set equal to their zero mean values, i.e.,

$G_{i}^{*}=G\left(0,0, \ldots, Z_{i}, \ldots, 0\right)$

$G_{i}=\frac{G_{i}^{*}}{G_{0}}$

At the zero means

$H=G$ and $\frac{\partial H}{\partial z_{i}}=\frac{\partial G}{\partial z_{i}}$

Substitution of $H$ for $G$ in eqn. (13) results in the following equation:

$E\left[G^{k}(Z)\right]=G_{0}^{k} \prod_{i=1}^{n} \int_{-\infty}^{+\infty} \phi\left(z_{i}\right) G_{i}^{k}\left(z_{i}\right) \mathrm{d} z_{i}$

The repeated application of the integration formula, eqn. (6), leads to the approximation of eqn. (19):

$E\left[G^{k}(\boldsymbol{Z})\right] \cong G_{0}^{k} \prod_{i=1}^{n}\left(\sum_{j=1}^{m_{i}} w_{j} G_{i}^{k}\left(z_{i j}\right)\right)=G_{0}^{k} \prod_{i=1}^{n} E\left[G_{i}^{k}\left(Z_{i}\right)\right]$ 
where $m_{i}$ is the number of points considered for variable $Z_{i}$. Equation (20) is the product integration formula for multiple independent standard normal variables.

\section{Non-product integration formula}

For a multiple independent standard normal vector $Z$, the probability density function, eqn. (12), can be further expressed as

$f_{Z}\left(z_{1}, z_{2}, \ldots, z_{n}\right)=\frac{1}{\sqrt{(2 \pi)^{n}}} \exp \left(-\frac{1}{2} z_{1}^{2}-\frac{1}{2} z_{2}^{2}-\ldots-\frac{1}{2} z_{n}^{2}\right)$

As in the one-dimensional case, substituting eqn. (21) into eqn. (2) and $z_{i}=\sqrt{2} u_{i}\left(\mathrm{~d} z_{i}=\sqrt{2} \mathrm{~d} u_{i}\right)$, eqn. (2) can be rewriten as

$E\left[G^{k}(Z)\right]=\frac{1}{\sqrt{\pi^{n}}} \int_{-\infty}^{+\infty} \ldots \int_{-\infty}^{+\infty} \exp \left(-u_{1}^{2}-u_{2}^{2}-\ldots-u_{n}^{2}\right) G^{k}(\sqrt{2} \boldsymbol{U}) \mathrm{d} u_{1} \ldots \mathrm{d} u_{n}$

The integration of eqn. (22) can be evaluated using various Gauss quadrature rules [16,17]:

$E\left[G^{k}(Z)\right] \cong \frac{1}{\sqrt{\pi^{n}}} \sum_{j=1}^{m} a_{j} G^{k}\left(\sqrt{2} u_{1 j}, \ldots, \sqrt{2} u_{n j}\right)$

where $\alpha_{j}$ and $\left(u_{1 j}, \ldots, u_{n j}\right)$ are widely available Gauss quadrature weigth factors and points $[16,17]$.

Substituting $z_{i j}=\sqrt{2} u_{i j}$ and $w_{j}=\alpha_{j} / \sqrt{\pi^{n}}$ into eqn. (23) the following non-product integration formula is obtained:

$E\left[G^{k}\left(Z_{1}, \ldots, Z_{n}\right)\right] \cong \sum_{j=1}^{m} w_{j} G^{k}\left(z_{1 j}, z_{2 j}, \ldots, z_{n j}\right)$

Typical weights $w_{j}$ and points $\left(z_{1 j}, z_{2 j}, \ldots, z_{n j}\right)$ in independent standard normal space are given in Table 2.

\section{Variables with joint distribution known}

The integration formulas for independent standard normal variables can be extended to functions of variables with any type of joint distribution known. The extension is based on the Rosenblatt transformation [15].

Let a set of $n$ random variables $X=\left(X_{1}, \ldots, X_{n}\right)$ have a joint $\mathrm{CDF}=F_{X}\left(x_{1}, x_{2}, \ldots, x_{n}\right)$, which can be given in terms of a sequence of conditional distribution functions:

$F_{X}(X)=F_{X_{1}}\left(x_{1}\right) F_{X_{2} \mid X_{1}}\left(x_{2}\right) \ldots F_{X_{n} \mid X_{1} \ldots X_{n-1}}\left(x_{n}\right)$

The original vector $\boldsymbol{X}$ can be transformed sequentially and componentwise, using the following equalities;

$$
\begin{aligned}
& \Phi\left(z_{1}\right)=F_{X_{1}}\left(x_{1}\right) \\
& \Phi\left(z_{2}\right)=F_{X_{2} \mid X_{1}}\left(x_{2}\right) \\
& \Phi\left(z_{3}\right)=F_{X_{3} \mid X_{1} X_{2}}\left(x_{3}\right) \\
& \vdots \\
& \Phi\left(z_{n}\right)=F_{X_{n} \mid X_{1} \ldots X_{n-1}}\left(x_{n}\right)
\end{aligned}
$$


TABLE 2

Examples of points and weights for non-product integration formulas

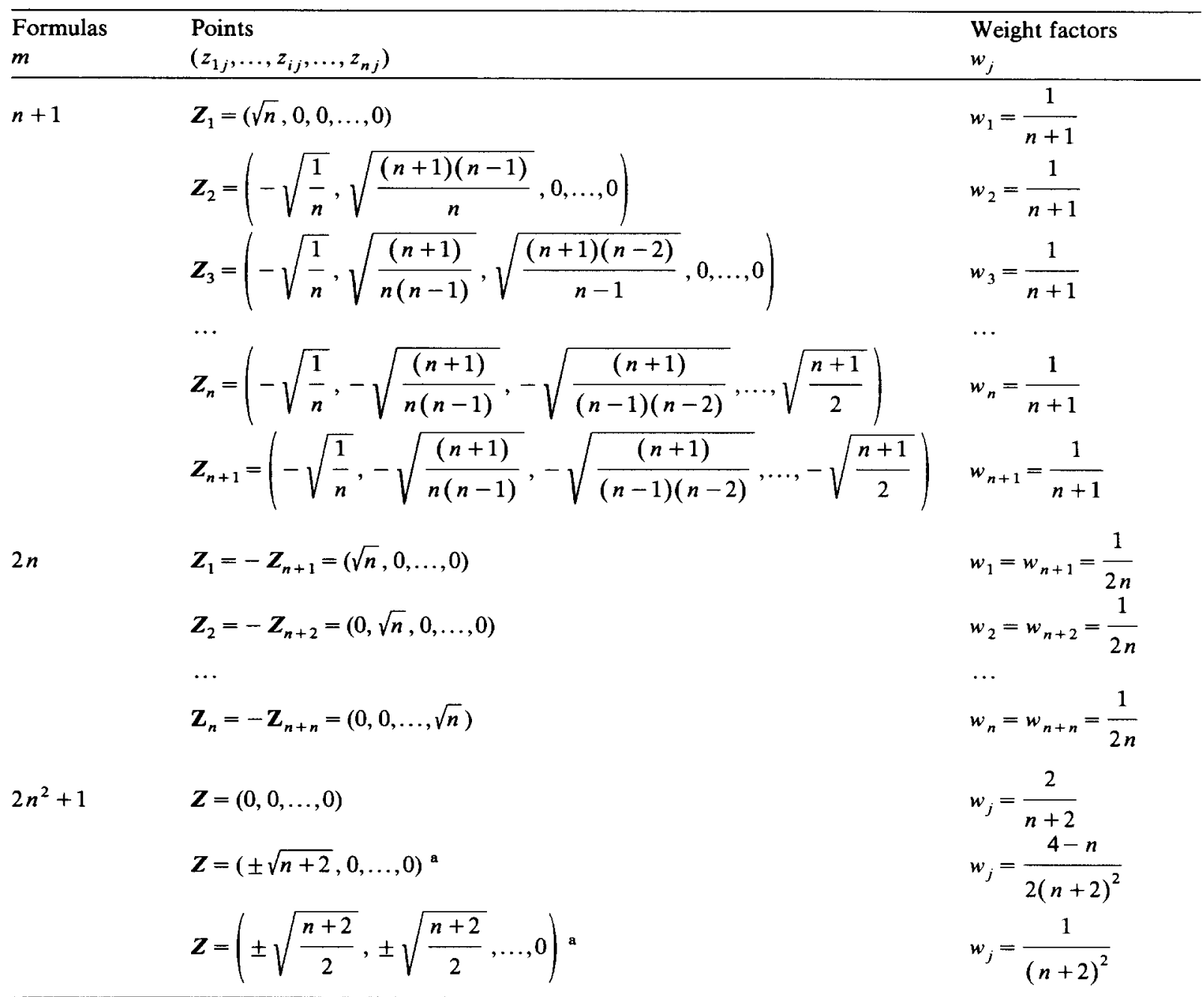

Points include all possible permutations of coordinates.

and hence

$x_{1}=F_{X_{1}}^{-1}\left[\Phi\left(z_{1}\right)\right]$

$x_{2}=F_{X_{2} \mid X_{1}}^{-1}\left[\Phi\left(z_{2}\right) \mid x_{1}\right]$

$x_{3}=F_{X_{3} \mid X_{1} X_{2}}^{-1}\left[\Phi\left(z_{3}\right) \mid x_{1} x_{2}\right]$

$x_{n}=F_{X_{n} \mid X_{1} \ldots X_{n-1}}^{-1}\left[\Phi\left(z_{n}\right) \mid x_{1} \ldots x_{n-1}\right]$ 
The Jacobian of the transformation is written as:

$J=\frac{\phi\left(z_{1}\right) \ldots \phi\left(z_{n}\right)}{f_{X}\left(x_{1}, \ldots, x_{n}\right)}$

By the above transformation, the integration of eqn. (2) over the non-normal space $\boldsymbol{X}$ is performed over the independent standard normal space $Z$ :

$$
\begin{aligned}
E\left[G^{k}(\boldsymbol{X})\right] & =\int_{-\infty}^{+\infty} \ldots \int_{-\infty}^{+\infty} f_{\boldsymbol{X}}\left(x_{1}, \ldots, x_{n}\right) G^{k}\left(x_{1}, \ldots, x_{n}\right) \mathrm{d} x_{1} \ldots \mathrm{d} x_{n} \\
& =\int_{-\infty}^{+\infty} \ldots \int_{-\infty}^{+\infty} f_{\boldsymbol{X}}\left(x_{1}, \ldots, x_{n}\right) G^{k}\left(x_{1}, \ldots, x_{n}\right) J \mathrm{~d} z_{1} \ldots \mathrm{d} z_{n} \\
& =\int_{-\infty}^{+\infty} \ldots \int_{-\infty}^{+\infty} \phi\left(z_{1}\right) \ldots \phi\left(z_{n}\right) G^{k}\left(x_{1}, \ldots, x_{n}\right) \mathrm{d} z_{1} \ldots \mathrm{d} z_{n}
\end{aligned}
$$

Equation (29) can be evaluated using either the product integration formula eqn. (20) or the non-product integration formula eqn. (24). Suppose eqn. (24) is used, then eqn. (29) becomes:

$E\left[G^{k}\left(X_{1}, \ldots, X_{n}\right)\right] \cong \sum_{j=1}^{m} w_{j} G^{k}\left(x_{1 j}, \ldots, x_{n j}\right)$

with

$x_{i j}=F_{X_{i} \mid X_{1} \ldots X_{i-1}}^{-1}\left[\Phi\left(z_{i j}\right) \mid x_{1 j} \ldots x_{(i-1) j}\right]$

where $i$ denotes the $i$ th variable and $j$ denotes $j$ th point; $w_{j}$ and $\left(z_{1 j}, \ldots, z_{i j}, \ldots, z_{n j}\right)$ can be obtained from Table 2.

Therefore, arbitrary distributions can also be handled by integration formulas for normal distributions provided that the conditional distribution functions can be produced. In principle, there are $n$ ! probabilistically equivalent transformations differing in the ordering of the variables. In general, not all of them have the same favorable numerical properties [18]. Therefore, in some problematic cases it is worthwhile to try one of the alternatives.

\section{Variables with marginal distributions known}

In many applications, the joint $\operatorname{PDF} f_{X}\left(x_{1}, \ldots, x_{n}\right)$ is often unknown, as complete statistical information on $\boldsymbol{X}$ is seldom available. In most situations the information available on $\boldsymbol{X}$ consists of only the mean vector $\bar{X}$, the correlation matrix $\boldsymbol{C}$, and the set of marginal CDF's, $F_{X_{i}}\left(x_{i}\right), i=$ $1, \ldots, n$. In this situation the integration formulas derived in the previous sections cannot be directly applied. To use the formaulas it is natural to seek a multivariate distribution model which itself is based on the normal distribution. In addition, the model sought must be consistent with the known marginals and the correlation matrix of the random vector $\boldsymbol{X}$. There are a large number of such models available in the literature [19]. The model used by Der Kiureghian and Liu [20] for reliability analysis under incomplete probability information is chosen for the current study.

First define $Y=\left(Y_{1}, Y_{2}, \ldots, Y_{n}\right)$ as joint standard normal variables by assigning a joint distribution to $X_{1}, \ldots, X_{n}$ :

$f_{X}\left(x_{1}, \ldots, x_{n}\right)=\phi_{n}\left(y_{1}, \ldots, y_{n}, C_{0}\right) \frac{f_{X_{1}}\left(x_{1}\right) \ldots f_{X_{n}}\left(x_{n}\right)}{\phi\left(y_{1}\right) \ldots \phi\left(y_{n}\right)}$ 
in which $f_{X_{i}}\left(\mathrm{x}_{\mathrm{i}}\right)=\mathrm{d} F_{X_{i}}\left(x_{i}\right) / \mathrm{d} x_{i}$ is the marginal PDF of $X_{i}$, and $\phi_{n}\left(\boldsymbol{Y}, \boldsymbol{C}_{0}\right)$ is the joint PDF of $n$-dimension normal variates with zero means, unit standard deviations, and correlation matrix $C_{0}$. The correlation coefficients $\rho_{0, i j}$ of $C_{0}$ are expressed in terms of the correlation coefficient $\rho_{i j}$ of $X_{i}$ and $X_{j}$ through the integration:

$$
\begin{aligned}
\rho_{i j} & =\int_{-\infty}^{\infty} \int_{-\infty}^{\infty}\left(\frac{x_{i}-\bar{X}_{i}}{\sigma_{i}}\right)\left(\frac{x_{j}-\bar{X}_{j}}{\sigma_{j}}\right) \phi_{2}\left(y_{i}, y_{j}, \rho_{0, i j}\right) \frac{f_{X_{i}}\left(x_{i}\right) f_{X_{j}}\left(x_{j}\right)}{\phi\left(y_{i}\right) \phi\left(y_{j}\right)} \mathrm{d} x_{i} \mathrm{~d} x_{j} \\
& =\int_{-\infty}^{\infty} \int_{-\infty}^{\infty}\left(\frac{x_{i}-\bar{X}_{i}}{\sigma_{i}}\right)\left(\frac{x_{j}-\bar{X}_{j}}{\sigma_{j}}\right) \phi_{2}\left(y_{i}, y_{j}, \rho_{0, i j}\right) \mathrm{d} y_{i} \mathrm{~d} y_{j}
\end{aligned}
$$

Equation (33) can be solved iteratively to find $\rho_{0, i j}$. To avoid such tedious calculations, approximate formulas for the ratio

$F=\rho_{0, i j} / \rho_{i j}$

are available in the literature $[20,21]$.

After establishing the multivariable distribution model, eqn. (32), the $k$ th moment of the function $G\left(X_{1}, \ldots, X_{n}\right)$ can be evaluated as the mathematical expectation of $G^{k}(X)$

$$
\begin{aligned}
E\left[G^{k}(\boldsymbol{X})\right] & =\int_{-\infty}^{+\infty} \cdots \int_{-\infty}^{+\infty} f_{\boldsymbol{X}}\left(x_{1}, \ldots, x_{n}\right) G^{k}(\boldsymbol{X}) \mathrm{d} x_{1} \ldots \mathrm{d} x_{n} \\
& =\int_{-\infty}^{+\infty} \cdots \int_{-\infty}^{+\infty} \phi_{n}\left(\boldsymbol{Y}, \boldsymbol{C}_{0}\right) \frac{f_{X_{1}}\left(x_{1}\right) \ldots f_{X_{n}}\left(x_{n}\right)}{\phi\left(y_{1}\right) \ldots \phi\left(y_{n}\right)} G^{k}(\boldsymbol{X}) \mathrm{d} \boldsymbol{X}
\end{aligned}
$$

The domain of the above integration can be converted from non-normal space $\boldsymbol{X}$ to the correlated standard normal space $\boldsymbol{Y}$ by the marginal transformation:

$Y_{i}=\Phi^{-1}\left[F_{X_{i}}\left(x_{i}\right)\right] \quad i=1,2, \ldots, n$

To use the integration formulas derived in previous sections the correlated normal space $Y$ must be mapped onto uncorrelated standard normal space $\boldsymbol{Z}$ by the linear transformation:

$\boldsymbol{Z}=\boldsymbol{L}_{0}^{-1} \boldsymbol{Y}$

where $\boldsymbol{L}_{0}=$ the lower triangular matrix obtained from Cholesky decomposition of $\boldsymbol{C}_{0}$.

Since eqns. (36) and (37) are two one-to-one transformations, their inverse transformations exist and therefore $\boldsymbol{Z}$ can be mapped to $\boldsymbol{Y}$, and then $\boldsymbol{Y}$ to $\boldsymbol{X}$

$$
\begin{aligned}
& \boldsymbol{Y}=\boldsymbol{L}_{0} \boldsymbol{Z} \\
& X_{i}=F_{X_{i}}^{-1}\left[\boldsymbol{\Phi}\left(y_{i}\right)\right]
\end{aligned}
$$

Combining eqn. (38) with eqn. (39) finally $Z$ is mapped to $X$ :

$\boldsymbol{X}=\boldsymbol{F}^{-1}\left[\Phi\left(\boldsymbol{L}_{0} Z\right)\right]$

The Jacobian of the combined transformation, eqn. (40), is obtained as::

$J=\frac{\phi\left(y_{1}\right) \ldots \phi\left(y_{n}\right)}{f_{X_{1}}\left(x_{1}\right) \ldots f_{X_{n}}\left(x_{n}\right)}\left|\boldsymbol{L}_{0}\right|$ 
where

$$
\left|\boldsymbol{L}_{0}\right|=\left|\begin{array}{ccc}
\frac{\partial y_{1}}{\partial z_{1}} & \cdots & \frac{\partial y_{1}}{\partial z_{n}} \\
\vdots & \ddots & \vdots \\
\frac{\partial y_{n}}{\partial z_{1}} & \cdots & \frac{\partial y_{n}}{\partial z_{n}}
\end{array}\right|
$$

By the transformation, eqn. (40), the integration, eqn. (35), finally is perfomed over the domain of the uncorrelated standard normal space:

$$
\begin{aligned}
\int_{-\infty}^{+\infty} & \ldots \int_{-\infty}^{+\infty} \phi_{n}\left(\boldsymbol{Y}, \boldsymbol{C}_{0}\right) \frac{f_{X_{1}}\left(x_{1}\right) \ldots f_{X_{n}}\left(x_{n}\right)}{\phi\left(y_{1}\right) \ldots \phi\left(y_{n}\right)} G^{k}(\boldsymbol{X}) \mathrm{d} x_{1} \ldots \mathrm{d} x_{n} \\
& =\int_{-\infty}^{+\infty} \ldots \int_{-\infty}^{+\infty} \phi_{n}\left(\boldsymbol{Y}, C_{0}\right) \frac{f_{X_{1}}\left(x_{1}\right) \ldots f_{X_{n}}\left(x_{n}\right)}{\phi\left(y_{1}\right) \ldots \phi\left(y_{n}\right)} G^{k}(\boldsymbol{X}) J \mathrm{~d} z_{1} \ldots \mathrm{d} z_{n} \\
& =\int_{-\infty}^{+\infty} \ldots \int_{-\infty}^{+\infty} \phi\left(z_{1}\right) \ldots \phi\left(z_{n}\right) G^{k}(X) \mathrm{d} z_{1} \ldots \mathrm{d} z_{n}
\end{aligned}
$$

The integration, eqn. (43), can be evaluated using either product or non-product integration formulas. Suppose the non-product formula eqn. (24) is used, then eqn. (43) is calculated by

$$
E\left[G^{k}(\boldsymbol{X})\right] \cong \sum_{j=1}^{m} w_{j} G^{k}\left(x_{1 j}, x_{2 j}, \ldots, x_{n j}\right)
$$

in which $\left(x_{1 j}, x_{2 j}, \ldots, x_{n j}\right)$ is obtained using eqns. (38) and (39):

$$
\begin{aligned}
& \left(\begin{array}{c}
y_{1 j} \\
\vdots \\
y_{n j}
\end{array}\right)=\left(\begin{array}{ccc}
L_{11} & \ldots & 0 \\
\vdots & L_{i i} & \vdots \\
L_{n 1} & \ldots & L_{n n}
\end{array}\right)\left(\begin{array}{c}
z_{1 j} \\
\vdots \\
z_{n j}
\end{array}\right) \\
& \left(\begin{array}{c}
x_{1 j} \\
\vdots \\
x_{n j}
\end{array}\right)=\left(\begin{array}{c}
F_{X_{1}}^{-1}\left[\Phi\left(y_{1 j}\right)\right] \\
\vdots \\
F_{X_{n}}^{-1}\left[\Phi\left(y_{n j}\right)\right]
\end{array}\right)
\end{aligned}
$$

where the first subscript $i$ denotes the $i$ th variable and the second subscript $j$ denotes the $j$ th point; $w_{j}$ and $\left(z_{1 j}, \ldots, z_{n j}\right)$ can be obtained from Table 2.

\section{NUMERICAL EXAMPLES}

Three examples are given to illustrate the use of the developed integration formulas. The application of the approach to bridge reliability analysis can be found in Ref. [22]. 


\section{Example 1. Single variable function}

A simple example is given here to show the use of integration formula eqn. (6) and eqn. (10). Consider a function of a single variable

$G(X)=X^{2}$

Case 1: Standard normal variable. Let $X$ be a standard normal variable denoted by $Z$ with zero mean and unit variance. The mean value of $G$ is calculated using three points. From eqn. (6):

$$
\begin{aligned}
\bar{G} & =E[G(Z)] \cong \sum_{j=1}^{3} w_{j} G\left(z_{j}\right) \\
& =\frac{1}{6}(-\sqrt{3})^{2}+\frac{4}{6}(0)^{2}+\frac{1}{6}(+\sqrt{3})^{2}=1
\end{aligned}
$$

The result is exact since in this case the mean of $G$ is equal to the variance of $Z\left(\sigma_{Z}^{2}=1\right)$.

Case 2: Non-normal variable. Assume that $X$ is a lognormally distributed random variable with cumulative distribution function (CDF):

$F_{X}(x)=\Phi\left(\frac{\ln x-2.283}{0.198}\right)$

In this case a four-point integration formula is used. The corresponding points and weight factors in standard normal space are taken from Table 2:

$\left(\begin{array}{l}z_{1} \\ z_{2} \\ z_{3} \\ z_{4}\end{array}\right)=\left(\begin{array}{l}-\sqrt{3+\sqrt{6}} \\ -\sqrt{3-\sqrt{6}} \\ +\sqrt{3-\sqrt{6}} \\ +\sqrt{3+\sqrt{6}}\end{array}\right)$ and $\left(\begin{array}{l}\omega_{1} \\ \omega_{2} \\ \omega_{3} \\ \omega_{4}\end{array}\right)=\frac{1}{12}\left(\begin{array}{l}3-\sqrt{6} \\ 3+\sqrt{6} \\ 3+\sqrt{6} \\ 3-\sqrt{6}\end{array}\right)$

The points in non-normal space are obtained using eqn. (11)

$x_{j}=F_{X}^{-1}\left(\Phi\left(z_{j}\right)\right)=\exp \left(2.283+0.198 z_{j}\right)$

$\left(\begin{array}{l}x_{1} \\ x_{2} \\ x_{3} \\ x_{4}\end{array}\right)=\left(\begin{array}{c}6.18 \\ 8.47 \\ 11.4 \\ 15.6\end{array}\right)$

The mean value of $G$ is calculated by using the integration formula eqn. (10),

$\bar{G} \cong \sum_{j=1}^{4} w_{j} G\left(x_{j}\right)=104$

The exact value of $\bar{G}$ is also 104 .

Calculations are also performed for $X$ with other types of distribution. The results are compared with the exact value. The comparisons are summarized in Table 3. The most accurate results are obtained for the integration formula eqn. (10). The mean value of $G$ is also evaluated using eqn. (10) with a different number of points. The results are shown in Table 4. From the 
TABLE 3

Two-point integration vs. types of random variables; $G=X^{2}$ with $\bar{X}=10, \sigma_{X}=2$

\begin{tabular}{|c|c|c|c|c|c|c|}
\hline Distribution & $x_{1}$ & $x_{2}$ & $\frac{x_{1}-\bar{X}}{\sigma_{X}}$ & $\frac{x_{2}-\bar{X}}{\sigma_{X}}$ & $\bar{G}$ & Error (\%) \\
\hline Normal & 8.00 & 12.00 & -1.000 & 1.000 & 104.00 & 0.00 \\
\hline Gamma & 8.01 & 11.99 & -0.995 & 0.995 & 103.95 & 0.05 \\
\hline Lognormal & 8.04 & 11.95 & -0.980 & 0.975 & 103.82 & 0.17 \\
\hline Beta & 7.96 & 12.04 & -1.020 & 1.020 & 104.16 & 0.16 \\
\hline Weibull & 7.98 & 12.00 & -1.010 & 1.000 & 104.04 & 0.04 \\
\hline Extreme Type I & 8.15 & 11.84 & -0.925 & 0.920 & 103.40 & 0.57 \\
\hline Extreme Type II & 8.35 & 11.57 & -0.825 & 0.785 & 102.59 & 1.36 \\
\hline
\end{tabular}

\section{TABLE 4}

Accuracy vs. number of integration points

\begin{tabular}{|c|c|c|c|c|c|}
\hline \multirow[t]{2}{*}{ Distribution } & \multicolumn{5}{|c|}{ Mean value of function: $G=X^{2}$} \\
\hline & 2 points & 3 points & 4 points & 5 points & 6 points \\
\hline Normal & 104.0000 & & & & \\
\hline Gamma & 103.9470 & 104.0000 & & & \\
\hline Lognormal & 103.8209 & 103.9968 & 104.0000 & & \\
\hline Beta & 104.1649 & 103.9932 & 104.0004 & 104.0000 & \\
\hline Weibull & 104.0421 & 104.0285 & 103.9944 & 104.0004 & 104.0000 \\
\hline Extreme Type I & 103.4037 & 103.9888 & 104.0032 & 103.9992 & 104.0000 \\
\hline Extreme Type II & 102.5860 & 103.6864 & 103.9470 & 103.9912 & 103.9992 \\
\hline
\end{tabular}

table one can see how many points are required in integration to obtain accurate results for different variables.

\section{Example 2. Multivariate function}

To illustrate the use of integration formulas for multivariates, consider the wind pressure acting on a window. The pressure is given by the formula

$G=\frac{1}{2} \gamma\left(X_{1}+X_{2}\right)^{2}$

where $G$ is the wind pressure, $\gamma$ is the density of air, $X_{1}$ is the basic wind speed, and $X_{2}$ is the gust wind speed. Consider $\gamma$ to be deterministic with a constant value of $2, X_{1}$ and $X_{2}$ to be random variables with $\bar{X}_{1}=10, \sigma_{X_{1}}=1, \bar{X}_{2}=20$, and $\sigma_{X_{2}}=1$.

Case 1: $X_{1}$ and $X_{2}$ with normal distribution. The independent standard normal variables are defined as

$$
\begin{aligned}
& Z_{1}=\frac{X_{1}-\bar{X}_{1}}{\sigma_{X_{1}}}=X_{1}-10 \\
& Z_{2}=\frac{X_{2}-\bar{X}_{2}}{\sigma_{X_{2}}}=X_{2}-20
\end{aligned}
$$


The wind pressure $G$ is further expressed in terms of the independent standard normal vector $Z$ :

$G=\left(Z_{1}+Z_{2}+30\right)^{2}$

Both product and non-product formulas are used to estimate the statistics of function $G$.

Using the product integration formula, eqn. (20), $G_{0}, G_{1}^{*}$, and $G_{2}^{*}$ are obtained as

$G_{0}=G(0,0)=900$

$G_{1}^{*}=G\left(Z_{1}, 0\right)=\left(Z_{1}+30\right)^{2}$

$G_{2}^{*}=G\left(0, Z_{2}\right)=\left(Z_{2}+30\right)^{2}$

From eqns. (15-17) the wind pressure $G$ is approximated by the product of two single-variable functions:

$G \cong H=900 \frac{\left(Z_{1}+30\right)^{2}}{900} \frac{\left(Z_{2}+30\right)^{2}}{900}$

From eqn. (20) the mean of the wind pressure acting on a window can be calculated by

$\bar{G} \cong 900\left(\sum_{j=1}^{m_{1}} w_{j} \frac{\left(z_{1 j}+30\right)^{2}}{900}\right)\left(\sum_{j=1}^{m_{2}} w_{j} \frac{\left(z_{2 j}+30\right)^{2}}{900}\right)$

If the two-point formula $\left(m_{1}=m_{2}=2\right)$ is used, then from Table 1,

$\left(\begin{array}{l}z_{11} \\ z_{12}\end{array}\right)=\left(\begin{array}{l}z_{21} \\ z_{22}\end{array}\right)=\left(\begin{array}{r}-1 \\ 1\end{array}\right) \quad$ and $\quad\left(\begin{array}{l}w_{1} \\ w_{2}\end{array}\right)=\left(\begin{array}{l}0.5 \\ 0.5\end{array}\right)$

Thus the mean of $G$ is

$\bar{G} \cong 900\left(\frac{29^{2}+31^{2}}{1800}\right)^{2}=902.0011$

The exact mean of $G$ is equal to 902 .

The non-product integration formula, eqn. (24), approximates the mean of $G$ by

$\bar{G} \cong \sum_{j=1}^{m} w_{j}\left(z_{1 j}+z_{2 j}+30\right)^{2}$

If a $(n+1)$-point formula is used, then from Table 2 the points and the weight factors are

$\left(\begin{array}{ll}z_{11} & z_{21} \\ z_{12} & z_{22} \\ z_{13} & z_{23}\end{array}\right)=\left(\begin{array}{cc}\sqrt{2} & 0 \\ -\sqrt{\frac{1}{2}} & \sqrt{\frac{3}{2}} \\ -\sqrt{\frac{1}{2}} & -\sqrt{\frac{3}{2}}\end{array}\right)$ and $\left(\begin{array}{l}w_{1} \\ w_{2} \\ w_{3}\end{array}\right)=\left(\begin{array}{c}\frac{1}{3} \\ \frac{1}{3} \\ \frac{1}{3}\end{array}\right)$

The mean value of $G, \bar{G}$, is calculated as

$\bar{G}=902$

This is the exact mean of $G$. 


\section{Case 2: $X_{1}$ and $x_{2}$ with joint PDF known.}

$$
\begin{aligned}
f_{X}\left(x_{1}, x_{2}\right)= & \frac{1}{\sqrt{2 \pi} \sigma_{X_{1}}} \exp \left(-\frac{1}{2}\left(\frac{x_{1}-\bar{X}_{1}}{\sigma_{X_{1}}}\right)^{2}\right) \frac{1}{\sqrt{2 \pi} \sigma_{\ln X_{2} \sqrt{1-\rho_{X_{1}, \ln X_{2}}^{2}}}} \frac{1}{x_{2}} \\
& \times \exp \left(-\frac{1}{2}\left(\frac{\ln x_{2}-\overline{\ln X_{2}}-\rho_{X_{1}, \ln X_{2}}\left(\sigma_{\ln X_{2}} / \sigma_{X_{1}}\right)\left(x_{1}-\bar{X}_{1}\right)}{\sigma_{\ln X_{2}} \sqrt{1-\rho_{X_{1}, \ln X_{2}}^{2}}}\right)^{2}\right)
\end{aligned}
$$

where

$$
\begin{aligned}
& \sigma_{\ln X_{2}}=\sqrt{\ln \left(1+V_{X_{2}}^{2}\right)} \\
& \overline{\ln X_{2}}=\ln \bar{X}_{2}-\frac{1}{2} \sigma_{X_{2}}^{2} \\
& \rho_{X_{1}, \ln X_{2}}=\frac{V_{X_{2}} \rho_{X_{1}, X_{2}}}{\sqrt{\ln \left(1+V_{X_{2}}^{2}\right)}}
\end{aligned}
$$

and

$$
\left[\begin{array}{ll}
\rho_{X_{1}, X_{1}} & \rho_{X_{1}, X_{2}} \\
\rho_{X_{2}, X_{1}} & \rho_{X_{2}, X_{2}}
\end{array}\right]=\left[\begin{array}{ll}
1 & 0.6 \\
0.6 & 1
\end{array}\right]
$$

The solution for the first two moments of $G$ proceeds as follows:

(1) Find conditional CDF's from the given joint distribution.

$$
\begin{aligned}
& F_{X_{1}}\left(x_{1}\right)=\Phi\left(\frac{x_{1}-\bar{X}_{1}}{\sigma_{X_{1}}}\right) \\
& F_{X_{2} \mid X_{1}}\left(x_{2} \mid x_{1}\right)=\Phi\left(\frac{\ln x_{2}-\overline{\ln X_{2}}-\rho_{X_{1}, \ln X_{2}}\left(\sigma_{\ln X_{2} / \sigma_{X_{1}}}\right)\left(x_{1}-\bar{X}_{1}\right)}{\sigma_{\ln X_{2}} \sqrt{1-\rho_{X_{1}, \ln X_{2}}^{2}}}\right)
\end{aligned}
$$

(2) Perform the Rosenblatt transformation using eqn. (27). In this example, the transformation can be obtained as follows,

$X_{1}=F_{X_{1}}^{-1}\left[\Phi\left(z_{1}\right)\right]=\bar{X}_{1}+\sigma_{X_{1}} Z_{1}$

$X_{2}=F_{X_{2} \mid X_{1}}^{-1}\left[\Phi\left(z_{2}\right) \mid x_{1}\right]=\exp \left(\overline{\ln X_{2}}+\rho_{X_{1}, \ln X_{2}}\left(\sigma_{\ln X_{2}} / \sigma_{X_{1}}\right)\left(x_{1}-\bar{X}_{1}\right)+\sigma_{\ln X_{2}} \sqrt{1-\rho_{X_{1}, \ln X_{2}}^{2}} Z_{2}\right)$

Using the given parameters the above two equations can be simplified as

$$
\begin{aligned}
& X_{1}=10+Z_{1} \\
& X_{2}=\exp \left(2.9956+0.03 Z_{1}+0.04 Z_{2}\right)
\end{aligned}
$$


(3) Select the points and weight factors in the standard normal space. If the $(n+1)$-point formula is used, then from Table 2 the points and the weight factors are

$\left(\begin{array}{ll}z_{11} & z_{21} \\ z_{12} & z_{22} \\ z_{13} & z_{23}\end{array}\right)=\left(\begin{array}{cc}\sqrt{2} & 0 \\ -\sqrt{\frac{1}{2}} & \sqrt{\frac{3}{2}} \\ -\sqrt{\frac{1}{2}} & -\sqrt{\frac{3}{2}}\end{array}\right)$ and $\left(\begin{array}{l}w_{1} \\ w_{2} \\ w_{3}\end{array}\right)=\left(\begin{array}{c}\frac{1}{3} \\ \frac{1}{3} \\ \frac{1}{3}\end{array}\right)$

(4) Calculate $\left(x_{1 j}, x_{2 j}\right)$ using eqn. (31)

$\left(\begin{array}{ll}x_{11} & x_{21} \\ x_{12} & x_{22} \\ x_{13} & x_{23}\end{array}\right)=\left(\begin{array}{rr}11.414 & 20.866 \\ 9.293 & 20.563 \\ 9.293 & 18.644\end{array}\right)$

(5) Calculate the first two moments of $G$ using eqn. (30)

$E[G(X)]=\sum_{j}^{3} \frac{1}{3} G\left(x_{1 j}, x_{2 j}\right)=904.484$

$E\left[G^{2}(X)\right]=\sum_{j}^{3} \frac{1}{3} G^{2}\left(x_{1 j}, x_{2 j}\right)=829821.3$

The exact value of $\bar{G}$ for this case is equal to 902.72 .

Case 3: $X_{1}$ and $X_{2}$ with marginal distributions known

$F_{X_{1}}\left(x_{1}\right)=\exp \left(-\exp \left(-1.283\left(x_{1}-9.55\right)\right)\right)$

$F_{X_{2}}\left(x_{2}\right)=\Phi\left(\frac{\ln x_{2}-2.996}{0.05}\right)$

and the correlation matrix

$C=\left[\begin{array}{ll}1 & 0.6 \\ 0.6 & 1\end{array}\right]$

The solution for the first two moments of $G$ proceeds as follows:

(1) Construct the correlation matrix $\boldsymbol{C}_{\mathbf{0}}$ by using the available formulas [20,21],

$C_{0}=\left[\begin{array}{ll}1 & 0.6158 \\ 0.6158 & 1\end{array}\right]$

(2) Determine $L_{0}$ by Cholesky decomposition of $C_{0}$ :

$\boldsymbol{L}_{0}=\left[\begin{array}{ll}1 & 0 \\ 0.6158 & 0.7879\end{array}\right]$

(3) Select normal integration formulas. Suppose the $(n+1)$ non-product formula is used,

$\left[\begin{array}{ccc}z_{11} & z_{12} & z_{13} \\ z_{21} & z_{22} & z_{23}\end{array}\right]=\left[\begin{array}{ccc}\sqrt{2} & -\frac{\sqrt{2}}{2} & -\frac{\sqrt{2}}{2} \\ 0 & \frac{\sqrt{3}}{2} & -\frac{\sqrt{3}}{2}\end{array}\right]$ 
(4) Calculate correlated standard normal vectors $Y$ using eqn. (45)

$$
\begin{aligned}
& \left(\begin{array}{l}
y_{1 j} \\
y_{2 j}
\end{array}\right)=\left(\begin{array}{ll}
1 & 0 \\
0.6158 & 0.7879
\end{array}\right)\left(\begin{array}{l}
z_{1 j} \\
z_{2 j}
\end{array}\right) \\
& {\left[\begin{array}{lll}
y_{11} & y_{12} & y_{13} \\
y_{21} & y_{22} & y_{23}
\end{array}\right]=\left[\begin{array}{lrr}
1.414 & -0.707 & -0.707 \\
0.871 & 0.530 & -1.40
\end{array}\right]}
\end{aligned}
$$

(5) Perform marginal transformation using eqn. (46)

$$
\begin{aligned}
& x_{1 j}=F_{X_{1}}^{-1}\left(\Phi\left(y_{1 j}\right)\right)=9.55-\frac{1}{1.283} \ln \left(-\ln \left(\Phi\left(y_{1 j}\right)\right)\right) \\
& x_{2 j}=F_{X_{2}}^{-1}\left(\Phi\left(y_{2 j}\right)\right)=\exp \left(2.995+0.05 y_{2 j}\right) \\
& {\left[\begin{array}{lll}
x_{11} & x_{12} & x_{13} \\
x_{21} & x_{22} & x_{23}
\end{array}\right]=\left[\begin{array}{lrr}
11.495 & 9.270 & 9.270 \\
20.887 & 20.534 & 18.645
\end{array}\right]}
\end{aligned}
$$

(6) Calculate the first two moments of $G$

$$
\begin{aligned}
& E[G]=\frac{1}{3} G\left(x_{11}, x_{21}\right)+\frac{1}{3} G\left(x_{12}, x_{22}\right)+\frac{1}{3} G\left(x_{13}, x_{23}\right)=905.4 \\
& E\left[G^{2}\right]=\frac{1}{3} G^{2}\left(x_{11}, x_{21}\right)+\frac{1}{3} G^{2}\left(x_{12}, x_{22}\right)+\frac{1}{3} G^{2}\left(x_{13}, x_{23}\right)=831930.5
\end{aligned}
$$

The exact mean of $G$ is 902.72 . The $0.2 \%$ error is due to the approximation in calculating the correlation coefficient $\rho_{0,12}$ and the nonlinearity of $G$ expressed in terms of independent standard variables. The error due to the nonlinearity of $G$ can be reduced by using integration formulas with more points.

\section{Example 3. Modeling of reinforced concrete section}

In this example the ultimate bending moment of the reinforced section shown in Fig. 3 is considered. This case was also studied by Ditlevsen [23]. The ultimate bending moment is

$$
M_{\mathrm{U}}=X_{1} X_{2} X_{3}-X_{4} \frac{X_{1}^{2} X_{2}^{2}}{X_{5} X_{6}}
$$

where $X_{1}$ is the area of reinforcement, $X_{2}$ the yield stress of the reinforcement, $X_{3}$ the effective depth of the reinforcement, $X_{4}$ a factor related to the stress-strain relationship of concrete, $X_{5}$

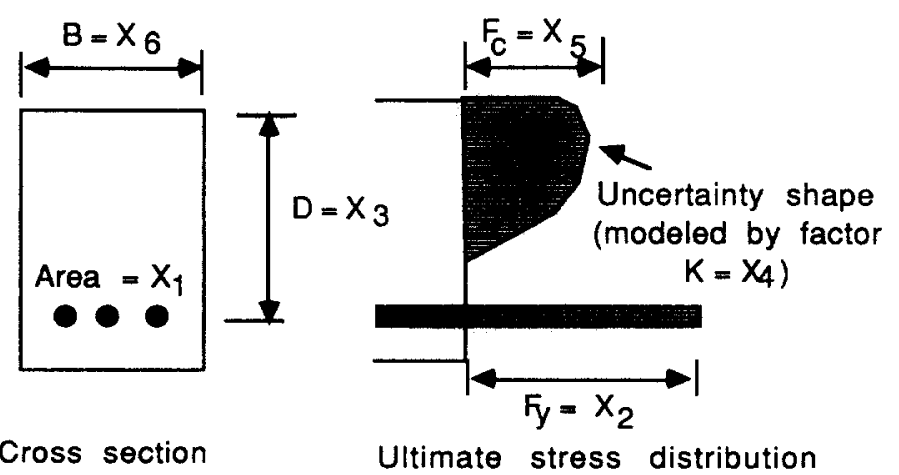

Fig. 3. State of ultimate stresses for reinforced concrete section. 
TABLE 5

Statistics of basic variables in Example 3

\begin{tabular}{lll}
\hline Variable & Mean value & Standard deviation \\
\hline$X_{1}$ & $1260 \mathrm{~mm}^{2}$ & $63 \mathrm{~mm}^{2}$ \\
$X_{2}$ & $250 \mathrm{~N} / \mathrm{mm}^{2}$ & $17.5 \mathrm{~N} / \mathrm{mm}^{2}$ \\
$X_{3}$ & $770 \mathrm{~mm}$ & $10 \mathrm{~mm}$ \\
$X_{4}$ & 0.55 & 0.055 \\
$X_{5}$ & $30 \mathrm{~N} / \mathrm{mm}^{2}$ & $4.5 \mathrm{~N} / \mathrm{mm}^{2}$ \\
$X_{6}$ & $250 \mathrm{~mm}$ & $5 \mathrm{~mm}$ \\
\hline
\end{tabular}

TABLE 6

Comparison of integration method with Monte Carlo method

\begin{tabular}{lll}
\hline Method & $\begin{array}{l}\bar{R}_{\mathrm{FM}}\left(\mathbf{1 0}^{\mathbf{6}}\right) \\
(\mathrm{N} \mathrm{mm})\end{array}$ & $V_{\mathrm{FM}}$ \\
\hline 3-point integration & 235 & 0.085 \\
5-point integration & 235 & 0.085 \\
100 simulations & 237 & 0.074 \\
500 simulations & 234 & 0.086 \\
5000 simulations & 235 & 0.084 \\
\hline
\end{tabular}

the maximum compressive strength of the concrete, and $X_{6}$ is the width of the beam. $\left(X_{1}, X_{2}, \ldots, X_{6}\right)$ are basic variables with lognormal distributions, and mutually uncorrelated. The mean values and standard deviations are given in Table 5.

The mean and coefficient of variation of $M_{\mathrm{U}}$ were calculated using 3- and 5-point product integration formulas, and also evaluated using Monte Carlo simulations. The results given in Table 6 indicate that integration formulas give results with good accuracy.

\section{CONCLUSIONS}

A set of numerical integration formulas were developed to compute the statistical parameters of a function of multiple random variables. The formula is a numerical procedure to estimate integrals using selected weights and points. The points and weights are predetermined in the independent standard normal variable space. The sample points in basic variable space are obtained by special transformations. The formulas were developed depending on the available statistical data about the basic variables.

\section{ACKNOWLEDGEMENTS}

The presented research has been sponosored by the National Science Foundation under Grant No. ECE-8413274, with Program Director John B. Scalzi, which is gratefully acknowledged. Thanks are due to Niels $\mathrm{C}$. Lind for his valuable suggestions and critical comments during his visits to the University of Michigan. 


\section{REFERENCES}

1 A.H.-S. Ang and C.A. Cornell, Reliability basis of structural safety and design, J. Struct. Div. ASCE, 103 (Sept. 1974) Proc. Paper 10777.

2 T.V. Galambos and M.K. Ravindra, Load and resistance factor design, J. Struct. Div. ASCE, ST9, Proc. Paper 14008 (Sept. 1978) 1335-1336.

3 B.R. Ellingwood, T.V. Galambos, J.G. MacGregor and C.A Cornell, Development of a probability based load criterion for American National Standard A58, Natl. Bur. Stand., Spec. Publ. 577, Washington, DC, June 1980.

4 N.C. Lind, The design of structural design norms, J. Struct. Mech., 1 (1973) 357-370.

5 O. Ditlevsen, Generalized second moment reliability index, J. Struct. Mech., ASCE, 7(4) (1979) $435-451$.

6 R. Rackwitz and B. Fiessler, Structural reliability under combined load sequences, Int. J. Comput. Struct., 9 (1978) 489-494.

7 H.O. Madsen, S. Krenk and N.C. Lind, Methods of Structural Safety, Prentice-Hall, Englewood Cliffs, NJ, 1986.

8 P. Thoft-Christensen and M.J. Baker, Structural Reliability Theory and Its Applications, Springer-Verlag, Berlin, 1982.

9 M.D. McKay, R.J. Beckman and W.J. Conover, A comparison of three methods for selecting values of input variables in the analysis of output from a computer code, Technometrics, 21(2) (May 1979) 239-245.

$10 \mathrm{~J}-\mathrm{H}$. Zhou and M.K. Ravindra, Application of Latin Hypercube Sampling in seismic PRA studies, Research Report, EQE Inc., Newport Beach, CA, August 1987.

11 E. Rosenblueth, Two-point estimates in probabilities, J. Appl. Math. Modeling, (May 1981).

12 N.C. Lind, Modeling of uncertainty in discrete dynamical systems, J. Appl. Math. Model., 7 (June 1983).

13 M.R. Corman, Structural resistance moments by quadrature, Structural Safety, 2 (1984) 73-81.

14 H.E. Salzer et al., Tables of the zero's and weight factors for first twenty Hermite polynomials, J. Res. Natl. Bur. Stand. Rp2294, 48 (1952) 111-116.

15 M. Rosenblatt, Remarks on a multivariate transformation, Ann. Math. Statist., 23(3) (September 1952$) 470-472$.

16 A.H. Stroud, Approximate Calculation of Multiple Integrals, Prentice-Hall, Englewood Cliffs, NJ, 1971

17 A.H. Stroud and D. Secrest, Approximate integration formulas for certain spherically symmetric regions, Math. of Computat., 17 (1963) 105-135.

18 K. Dolinski, First-order second-moment approximation of structural systems: Critical review and alternative approach, Structural Safety, 1(3) (1983) 211-231.

19 N.L. Johnson and S. Kotz, Distributions in Statistics-Continuous Multivariate Distributions, John Wiley \& Sons, New York, NY, 1976.

20 A. Der Kiureghian and P-L. Liu, Structural reliability under incomplete probability information, J. Eng. Mech., ASCE, 112 (January 1986) 85-104.

21 R.M. Bennett and E-K. Ko, Reliability of structures against progressive collapse, Research Series No. 40, The University of Tennessee, Department of Civil Engineering, October 1986.

22 J-H. Zhou and A.S. Nowak, Application of normal integration formulas to bridge reliability analysis, Proceedings of Structures Congress '87, ASCE, Vol. 4, Orlando, FL, 1987.

23 O. Ditlevsen, Uncertainty Modeling, McGraw-Hill, New York, NY, 1981, pp. 101-103. 